\title{
Variação sazonal da estrutura da comunidade de algas perifíticas em Panicum repens em um reservatório raso
}

Seasonal variation in the periphytic algal community structure on Panicum repens in a shallow reservoir

\author{
Mayara Ribeiro Casartelli ${ }^{1}$ \& Carla Ferragut ${ }^{1,2}$
}

\begin{abstract}
Resumo
Este estudo avaliou a estrutura da comunidade de algas perifíticas em Panicum repens em pontos pluriespecíficos de macrófitas em reservatório raso (Lago das Ninféias, São Paulo, Brasil). O objetivo foi avaliar as mudanças na biomassa, abundância e composição espécies, bem como identificar as variáveis ambientais mais relacionadas à variação dos atributos estruturais da comunidade. Pontos de amostragem com a presença de três e quatro espécies de macrófitas $(n=3)$ foram selecionados aleatoriamente $(04 / 2010$, 06/2010,11/2010,02/2011). Foram analisadas variáveis climáticas, abióticas da água, cobertura de macrófitas e atributos estruturais do perifíton. Bacillariophyceae e Chrysophyceae foram as classes algais mais abundantes e Frustulia crassinervia e Chromulina elegans as espécies mais representativas, respectivamente. Os maiores valores de biomassa e densidade algal e o menor índice autotrófico foram encontrados no verão, evidenciando o maior desenvolvimento da comunidade algal. Os nossos resultados mostraram que as mudanças na estrutura do perifíton foram determinadas primariamente pela sazonalidade. Concluiu-se que a abundância, biomassa e composição de algas perifíticas mudaram fortemente entre as estações do ano e que, a disponibilidade de luz e de nutrientes, particularmente o fósforo, foram as variáveis ambientais mais associadas às mudanças estruturais da comunidade durante o período de estudo.
\end{abstract}

Palavras-chave: biomassa, composição de espécies, densidade de algas, perifíton.

\begin{abstract}
This study evaluated the periphytic algal community structure on Panicum repens at macrophytes pluriespecifics sites in shallow reservoir (Ninféias Reservoir, São Paulo, Brazil). The aim was to evaluate changes in species composition and abundance, as well as identify the environmental factors most related to the variation of the periphyton structural attributes. Sampling sites with presence of three and four macrophytes species $(n=3)$ were randomly selected in seasons $(04 / 2010,06 / 2010,11 / 2010,02 / 2011)$. Climate and water variables, cover macrophyte and periphyton structural attributes were analyzed. Bacillariophyceae and Chrysophyceae were the most abundant algal class and Frustulia crassinervia and Chromulina elegans were the most representative species. The highest biomass and algal density and the lowest autotrophic index were found in summer, showing the further development of the algal community. Our results showed that changes in the periphyton structure on $P$. repens were determined primarily by seasonality. We conclude that periphyton abundance, biomass and composition changed greatly between the seasons and the light and nutrients availability, particularly phosphorus, were the variables most associated with the community structural changes during the study period. Key words: biomass, periphyton, species composition, algal density, perifíton.
\end{abstract}

\section{Introdução}

As algas perifíticas têm importante papel no funcionamento de lagos e reservatórios rasos, pois atuam como importante produtor primário e fonte de alimento para os outros níveis tróficos (Vadeboncoeur \& Steinman 2002). A estrutura e o funcionamento do perifíton nos ecossistemas aquáticos são influenciados direta

${ }^{1}$ Instituto de Botânica, Núcleo de Pesquisa em Ecologia, Av. Miguel Stéfano 3687, Água Funda, 04301-902, São Paulo, SP, Brasil.

${ }^{2}$ Autor para correspondência: carlaferragut@yahoo.com.br 
ou indiretamente por inúmeros fatores abióticos e bióticos (Stevenson 1997). Logo, a estrutura da comunidade de algas perifíticas pode mudar em função das condições ambientais, principalmente em relação à disponibilidade de luz e nutrientes, temperatura, turbulência e de substrato para a colonização (Liboriussen \& Jeppesen 2009; Vadeboncoeur \& Steinman 2002). Os fatores abióticos, principalmente a concentração de nutrientes na água, são considerados bons preditores da abundância e da composição de espécies de algas perifíticas em lagos e reservatórios tropicais (Algarte et al. 2006; Vercellino \& Bicudo 2006). Apesar do aumento dos estudos sobre o perifíton nas últimas décadas (Larned et al. 2010), o padrão de variação da comunidade perifítica em escala espacial e temporal é ainda um desafio para os pesquisadores.

Entre os substratos vivos, as macrófitas aquáticas são substratos bastante favoráveis para o desenvolvimento do perifíton, pois podem fornecer ampla área para colonização e, ainda, podem disponibilizar nutrientes a partir dos processos de excreção, senescência e decomposição (Burkholder 1996). Contudo, algumas espécies de macrófitas podem atuar negativamente sobre o desenvolvimento do perifíton, pois podem liberar substâncias alelopáticas (Erhard \& Gross 2006) e reduzir a disponibilidade de luz por meio de sombreamento (Grimshaw et al. 1997). O complexo perifíton-macrófita tem grande importância nos ecossistemas aquáticos, pois sintetiza grande quantidade de matéria orgânica (Wetzel 1990), altera a disponibilidade de nutrientes do meio (Burkholder \& Wetzel 1990) e, ainda, pode funcionar como um sistema natural de filtração (Kiss et al. 2003). Em nível ecossistêmico, a influência das macrófitas aquáticas sobre as alterações da qualidade da água é grandemente acrescida pelo desenvolvimento do perifíton em suas partes submersas (Lalonde \& Downing 1991; Kiss et al. 2003). Desta forma, a investigação sobre o desenvolvimento da comunidade de algas perifíticas em macrófitas pode contribuir para o maior entendimento da relação perifíton-macrófita e, consequentemente, do papel deste complexo no ecossistema.

A macrófita emergente Panicum repens L. é uma espécie introduzida que se expande nas áreas inundáveis do Pantanal Mato-Grossense e reservatórios do rio Paraná, principalmente em locais perturbados, substituindo espécies nativas
(Pott \& Pott 2003; Agostinho et al. 2005). Hanlon \& Langeland (2000) relataram que a invasora $P$. repens ("torpedograss") substituiu cerca de 6.000 hectares de espécies nativas dos 40.000 hectares da região litorânea do Lago Okeechobee (Flórida). Apesar da problemática em relação ao gênero Panicum, poucos estudos avaliaram a comunidade de algas perifíticas aderidas a esta macrófita, a qual visivelmente apresenta grande quantidade de biomassa perifítica em suas partes submersas. No Brasil, estudos relataram a elevada produção primária e a alta diversidade de espécies de algas perifíticas em Panicum rivulare Trin. (Bicudo et al. 1995; Martins \& Fernandes 2007) e em espécies de gramíneas (Cetto et al. 2004). Por outro lado, estudos observacionais e experimentais relataram a redução do desenvolvimento do perifíton em densos bancos de Panicum repens do Lago Okeechobee na Flórida (Grimshaw et al. 1997). Recentemente, Rodusky et al. (2013) encontrou biomassa perifítica maior em $P$. repens do que na macrófita nativa Eleocharis cellulosa no Lago Okeechobee (USA), além da elevada abundância de cianobactérias. Apesar dos inúmeros estudos sobre o controle e manejo dessa macrófita (Hanlon \& Langeland 2000; Hanlon \& Brady 2005), pouco se conhece sobre a relação perifíton-Panicum.

Considerando a importância do conhecimento do perifíton e do complexo perifíton-macrófita nos ecossistemas aquáticos, o presente estudo avaliou a estrutura da comunidade de algas perifíticas em Panicum repens em um reservatório raso. Este estudo foi realizado em pontos com diferentes números de espécies de macrófitas aquáticas, pois a riqueza de macrófitas pode influenciar a biomassa e o conteúdo de fósforo do perifíton (Engelhardt \& Ritchie 2001). O principal objetivo foi avaliar a variação sazonal da abundância, composição e diversidade de espécies de algas perifíticas, bem como identificar as variáveis ambientais mais relacionadas à variação dos atributos estruturais da comunidade durante o período de estudo. De modo geral, o presente estudo pretende contribuir para o maior entedimento da relação perifíton-macrófitas.

\section{Material e Métodos}

Área de estudo

O estudo foi realizado no Lago das Ninféias (233' S, 46³7’ W), o qual está localizado em área preservada de remanescente de Mata Atlântica dentro da área urbana da cidade de São Paulo, o Parque Estadual das Fontes do Ipiranga (PEFI). É 
um reservatório pequeno, raso e mesotrófico com área de $5.433 \mathrm{~m}^{2}$, profundidade máxima de 3,6 m, média de 1,32 m e tempo de residência de 7,2 dias e, ainda, caracteriza-se pela elevada cobertura de macrófitas aquáticas (Bicudo et al. 2002). A amostragem foi realizada na parte mais rasa da região litorânea, a qual apresenta profundidade média de 0,5 m. A área de estudo é caracterizada pela ocorrência de um período climático seco (março a agosto) e chuvoso (setembro a fevereiro). Durante o período de estudo, os valores médios mensais da precipitação pluviométrica acumulada e da temperatura foram, respectivamente, os seguintes: outono, $180,5 \mathrm{~mm}$ e $23,4{ }^{\circ} \mathrm{C}$ (abril); inverno, 89,7 $\mathrm{mm}$ e $20,1{ }^{\circ} \mathrm{C}$ (julho); primavera, $69,1 \mathrm{~mm}$ e $17,1{ }^{\circ} \mathrm{C}$ (novembro); verão, $208 \mathrm{~mm}$ e 23,9 ${ }^{\circ} \mathrm{C}$ (janeiro) <http://www.estacao.iag.usp.br/ boletim.php>.

\section{Delineamento amostral e variáveis analisadas}

A amostragem das variáveis físicas e químicas da água e do perifíton em Panicum repens foi realizada no outono (27/04/2010), inverno (28/07/2010), primavera (04/11/2010) e verão (14/02/2011). O estudo apresentou um total de 24 unidades amostrais na zona litorânea do Lago das Ninféias. O perifíton foi amostrado nas partes submersas do colmo de Panicum repens, que é uma macrófita emergente introduzida e, popularmente, conhecida como capim-furachão.

A amostragem foi realizada em três pontos com a presença de Panicum repens (emergente), Nymphaea spp. (enraizada de folhas flutuantes) e Utricularia foliosa L. (flutuante livre) e em dois pontos com a presença de Panicum repens, Nymphaea spp., Utricularia foliosa L. e Eleocharis acutangula (Roxb.) Schult (emergente). Os pontos com a presença de 3 e 4 espécies de macrófitas foram designados de $3 \mathrm{M}$ e 4M, respectivamente.

Amostras da subsuperfície da água foram coletadas para determinação das variáveis físicas, químicas e biológicas. Foram analisadas as seguintes variáveis abióticas: temperatura (termômetro), transparência da água (profundidade do disco de Secchi), radiação subaquática (luxímetro Li-Cor LI-250A), condutividade elétrica (condutivímetro Digimed), oxigênio dissolvido (Golterman et al. 1978), alcalinidade (Golterman \& Clymo 1971), pH (potenciômetro Digimed), formas de carbono inorgânico dissolvido ( $\mathrm{CO}_{2}$ livre), nitrito e nitrato (N-NO ${ }_{2}^{-}$e N-NO ${ }_{3}^{-}$; Mackeret et al. 1978), amônio
(N-NH ${ }_{4}^{+}$; Solorzano 1969), ortofosfato $\left(\mathrm{P}^{-} \mathrm{PO}_{4}^{-}\right)$ e fósforo total dissolvido (PDT; Strickland \& Parsons 1960), nitrogênio total (NT) e fósforo total (PT) (Valderrama 1981) e sílica solúvel reativa (Golterman et al. 1978). O nitrogênio inorgânico dissolvido (NID) refere-se à soma das concentrações de nitrogênio na forma de amônio, nitrito e nitrato.

O material perifítico foi retirado do colmo por meio de raspagem com escova de cerdas macias e jatos de água destilada. O comprimento e a largura de cada parte do colmo foram medidos para obtenção da área de colonização. Amostras de perifíton foram filtradas em filtro de fibra de vidro Whatman GF/F para determinação da clorofila-a e da massa seca livre de cinzas. A extração da clorofila-a (corrigida da feofitina) do perifíton foi feita utilizando etanol (90\%), segundo Marker et al. (1980) e Sartory \& Grobblelar (1984). A determinação da massa seca (MS) e massa seca livre de cinzas (MSLC) foi feita através de método gravimétrico e numérico (APHA 2005). A razão entre MSLC e a clorofila- $a$, Índice Autotrófico (IA), foi calculado para a avaliação da participação dos componentes autotróficos e heterotróficos no perifíton. Este índice apresenta limite teórico de 200, assim, valores superiores a 200 indicam predominância de heterótrofos e valores inferiores de autótrofos (APHA 2005).

A análise taxonômica da comunidade de algas perifíticas foi realizada em microscópio binocular Zeiss Axioplan 2 a partir de amostras fixadas com solução de formalina 4\%. Lâminas permanentes foram feitas para a identificação das diatomáceas (Battarbee 1986). Para a identificação das espécies utilizou-se literatura especializada e local (e.g. Bicudo et al. 2003; Bicudo 2004; Araújo \& Bicudo 2006). Amostras foram fixadas em lugol acético para contagem, a qual foi realizada em microscópio invertido Zeiss Axiovert (400x). A contagem foi realizada em transectos e o limite de contagem foi estabelecido de acordo com a curva de rarefação de espécies e até atingir 100 indivíduos da espécie mais abundante. Considerou-se como dominante a espécie com densidade superior a 50\% da densidade total da amostra. Em cada unidade amostral, as espécies com densidade relativa igual ou superior a $1 \%$ foram consideradas descritoras da comunidade de algas perifíticas. 
Tratamento dos dados

Para análise descritiva dos dados bióticos e abióticos utilizou-se medidas de tendência central (média aritmética) e de dispersão dos dados, como desvio padrão (Dp) e coeficiente de variação (CV).

Análise de Correspondência Canônica (CCA) foi realizada para determinar se a variância nos dados da comunidade de algas perifíticas poderia ser explicada pelas variáveis ambientais. A matriz principal foi composta pelas espécies com densidade relativa superior a $1 \%$ da densidade total de cada amostra e a matriz secundária foi composta por 5 variáveis abióticas, que foram selecionadas com base na PCA das variáveis limnológicas. As variáveis abióticas de maior correlação de Pearson com os eixos 1 e 2 foram selecionadas para CCA e as variáveis que poderiam causar multicolinearidade foram excluídas (Legendre \& Legendre 2012). A análise foi realizada a partir de dados transformados por logaritmo $(\log x+1)$ no programa PC-ORD 6.0 para Windows (McCune $\&$ Mefford 2011).

\section{Resultados}

A Tabela 1 resume as condições limnológicas nos pontos 3M e 4M em cada estação do ano. As condições limnológicas nestes dois pontos no outono e no inverno caracterizaram-se pela alta disponibilidade $\mathrm{N}$, sendo registradas as maiores concentrações de NT e nitrato no outono. Por sua vez, na primavera e no verão detectou-se os maiores valores de PDT e pH. Considerando a pequena profundidade dos pontos de amostragem $(0,4-0,8 \mathrm{~m})$, a disponibilidade de luz foi sempre elevada, pois a zona eufótica sempre chegou até o fundo do reservatório. Contudo, a transparência e a porcentagem de radiação subaquática apresentaram os maiores valores na primavera e no verão nos pontos $3 \mathrm{M}$ e $4 \mathrm{M}$.

Os maiores valores de cobertura de Panicum repens foram encontrados no verão, contribuindo, em média, com $23 \%$ e $17 \%$ para cobertura total nos pontos $3 \mathrm{M}$ e $4 \mathrm{M}$, respectivamente (Fig. 1). A cobertura total de macrófitas apresentou os maiores valores na primavera e no verão nos pontos 3M e 4M, sendo Nymphaea a macrófita de maior representatividade $(23,7 \%$ a $54,7 \%)$. A cobertura total apresentou pequena diferença entre os pontos $3 \mathrm{M}$ e $4 \mathrm{M}(4-13 \%)$. Apenas no verão ocorreu elevada abundância de Nymphaea spp e Utricularia foliosa (3M: 38\% e 35\%; 4M: 25 e $21 \%$, respectivamente).
Na comunidade de algas perifíticas em Panicum repens foram identificados 167 táxons, os quais foram distribuídos em nove classes: Bacillariophyceae (19 táxons), Chlorophyceae (73), Chrysophyceae (5), Cryptophyceae (5), Cyanobacteria (10), Dinophyceae (2), Euglenophyceae (7), Oedogoniophyceae (2) e Zygnemaphyceae (44).

Os atributos estruturais da comunidade de algas perifíticas em Panicum repens variaram grandemente entre as estações do ano nos pontos $3 \mathrm{M}$ e $4 \mathrm{M}$ (Fig. 2a-d). Em ambos os pontos, os maiores valores de clorofila- $a$ e densidade total foram registrados no verão. Os maiores valores de MSLC ocorreram nos pontos 3M e 4M no outono e inverno. Nestas estações do ano, o IA (MSCL:clorofila- $a$ ) revelou predominância do componente heterotrófico no perifíton. O coeficiente de variação dos dados de clorofila- $a$ $(3 \mathrm{M}=104 \% ; 4 \mathrm{M}=64 \%)$, densidade total $(3 \mathrm{M}=$ $72 \% ; 4 \mathrm{M}=12 \%)$ e $\mathrm{MSLC}(3 \mathrm{M}=57 \%$; $4 \mathrm{M}=30 \%)$ foi sempre maior nos pontos $3 \mathrm{M}$ do que nos $4 \mathrm{M}$.

Em termos de densidade algal, Bacillariophyceae, Chlorophyceae e Chrysophyceae apresentaram elevada participação na estrutura do perifíton nos pontos $3 \mathrm{Me} 4 \mathrm{M}$ em todas as estações do ano (Fig. 3). Nos pontos 3M, o perifíton apresentou dominância de diatomáceas no outono e no inverno e elevada densidade relativa de Chrysophyceae na primavera e no verão. Nos pontos $4 \mathrm{M}$, a densidade relativa de Bacillariophyceae e Chrysophyceae apresentou pequena flutuação entre as estações do ano. O coeficiente de variação da densidade de Bacillariophyceae e de Chrysophyceae foi elevado entre as estações do ano (CV: $3 \mathrm{M}=58-127 \%$; $4 \mathrm{M}=12-52 \%)$.

Considerando a densidade relativa das espécies de algas perifíticas mais abundantes ( $>10 \%$ da densidade total), verificou-se a presença constante de três espécies nos pontos $3 \mathrm{M}$ e $4 \mathrm{M}$ durante o período de estudo, foram elas: Frustulia crassinervia (Bréb.) Lange-Bert. \& Krammer, Gomphonema gracile Ehrenb. (Bacillariophyceae) e Chromulina cf. elegans Doflein (Chrysophyceae; Fig. 4). Nos pontos 3M, F. crassinervia foi a espécie mais abundante no outono e inverno e Chromulina cf. elegans na primavera e no verão. Diferentemente nos pontos 4M, Chromulina cf. elegans foi a espécie mais abundante em todas as estações do ano (38,6-39\%). Destaca-se o aumento da densidade relativa de Gomphonema gracile e Navicula cryptotenella (Bacillariophyceae) nos pontos 3M e 4M no verão. 


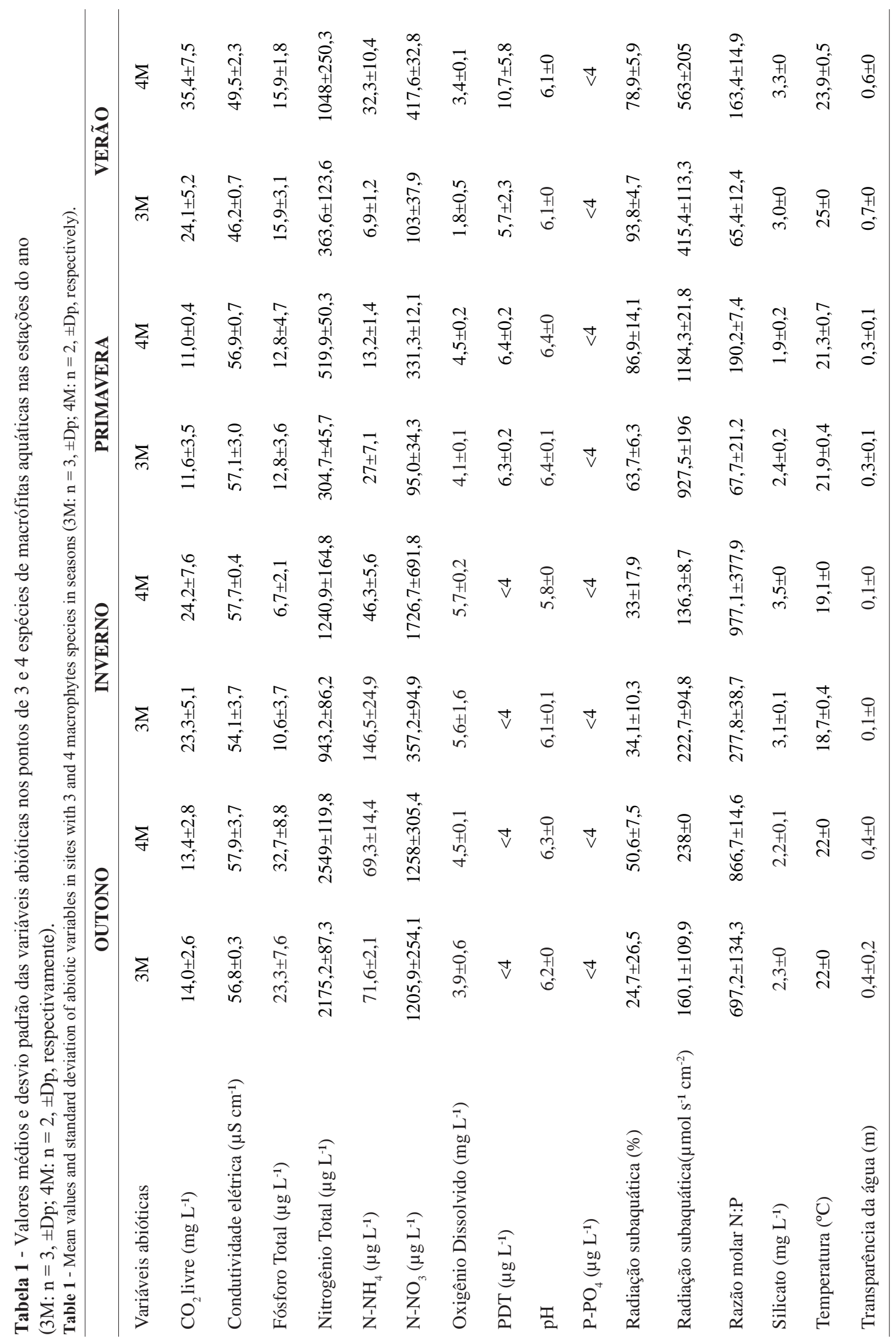




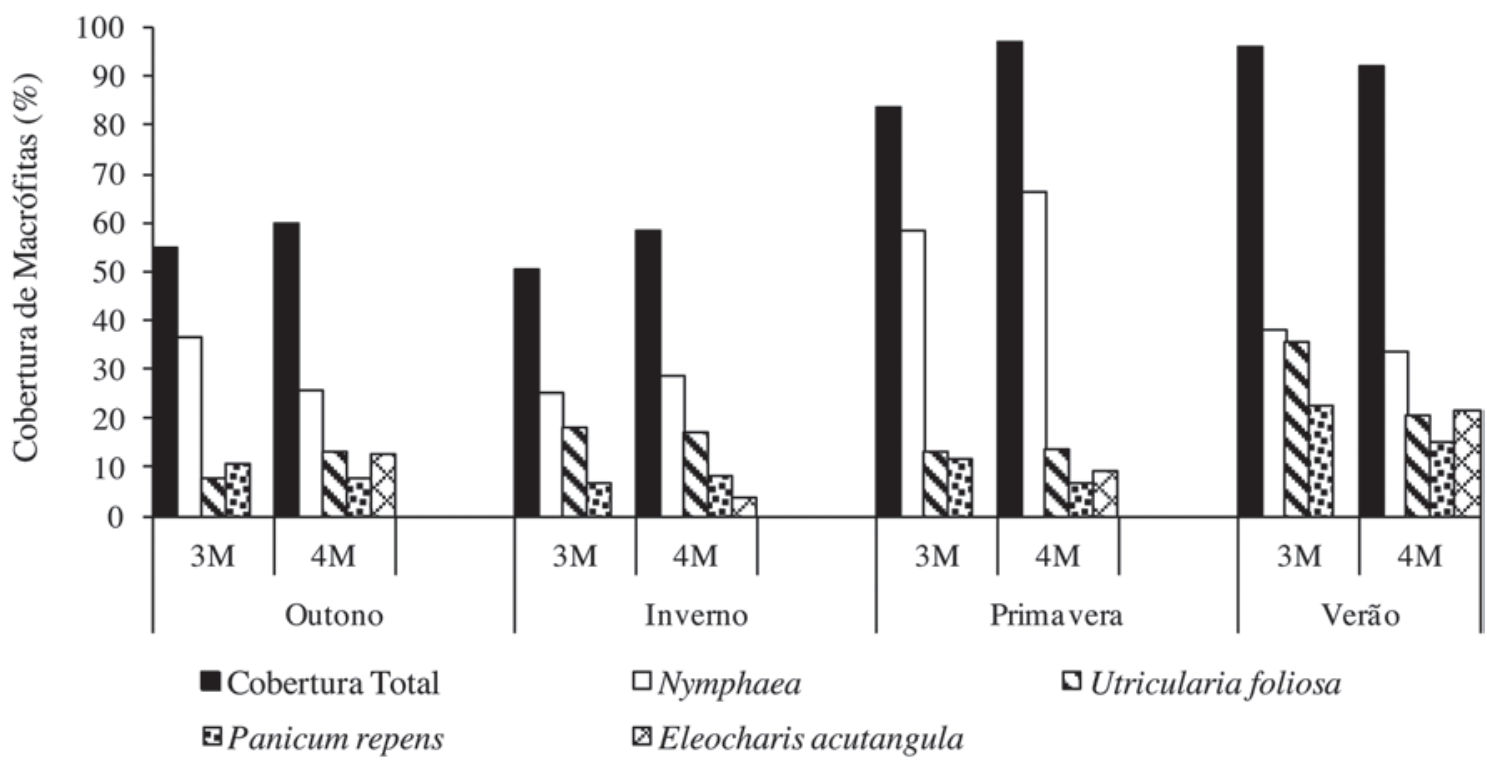

Figura 1 - Variação sazonal da cobertura de espécies de macrófitas $(n=3)$ nos pontos de amostragem nas estações do ano (3M, pontos 3 espécies de macrófitas; 4M, pontos 4 espécies).

Figure 1 - Seasonal variation of macrophyte species cover at sampling sites in seasons (3M, sites with 3 macrophytes species; 4M, sites with 4 species).
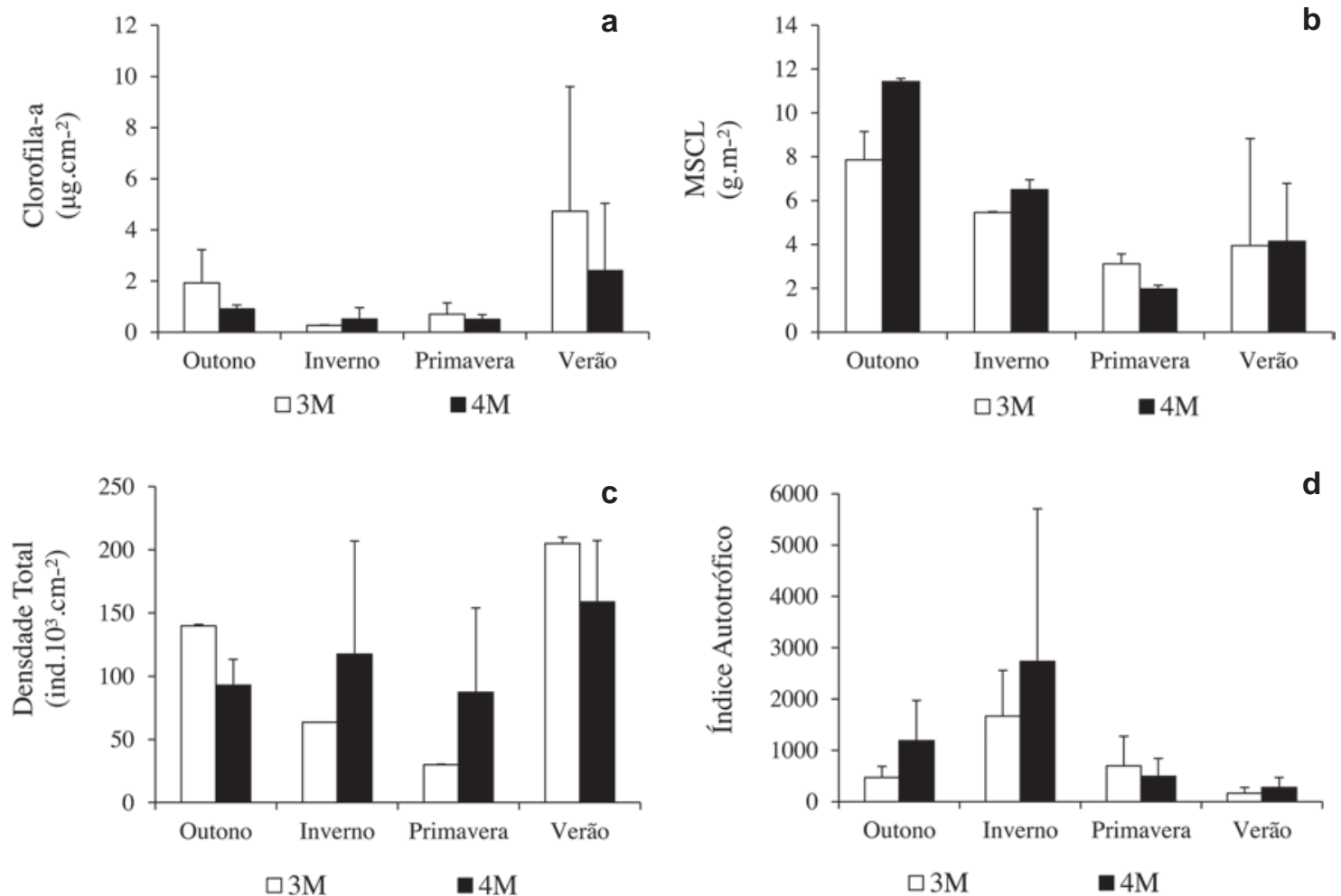

Figura 2 - Biomassa algal (a), massa seca livre de cinzas (MSLC, b), densidade total de algas (c) e Índice Autotrófico (d) da comunidade perifítica em Panicum repens nos pontos com presença de 3 e 4 espécies de macrófitas aquáticas nas estações do ano (3M: $n=3, \pm D p ; 4 M: n=2, \pm D p$, respectivamente).

Figure 2 - Periphytic algal biomass (a), ash free dry mass (AFDM, b), total density (c) and Autotrophic Index (d) on Panicum repens at sites with 3 and 4 macrophytes species in seasons (3M: $n=3, \pm D p ; 4 M: n=2, \pm D p$, respectively). 


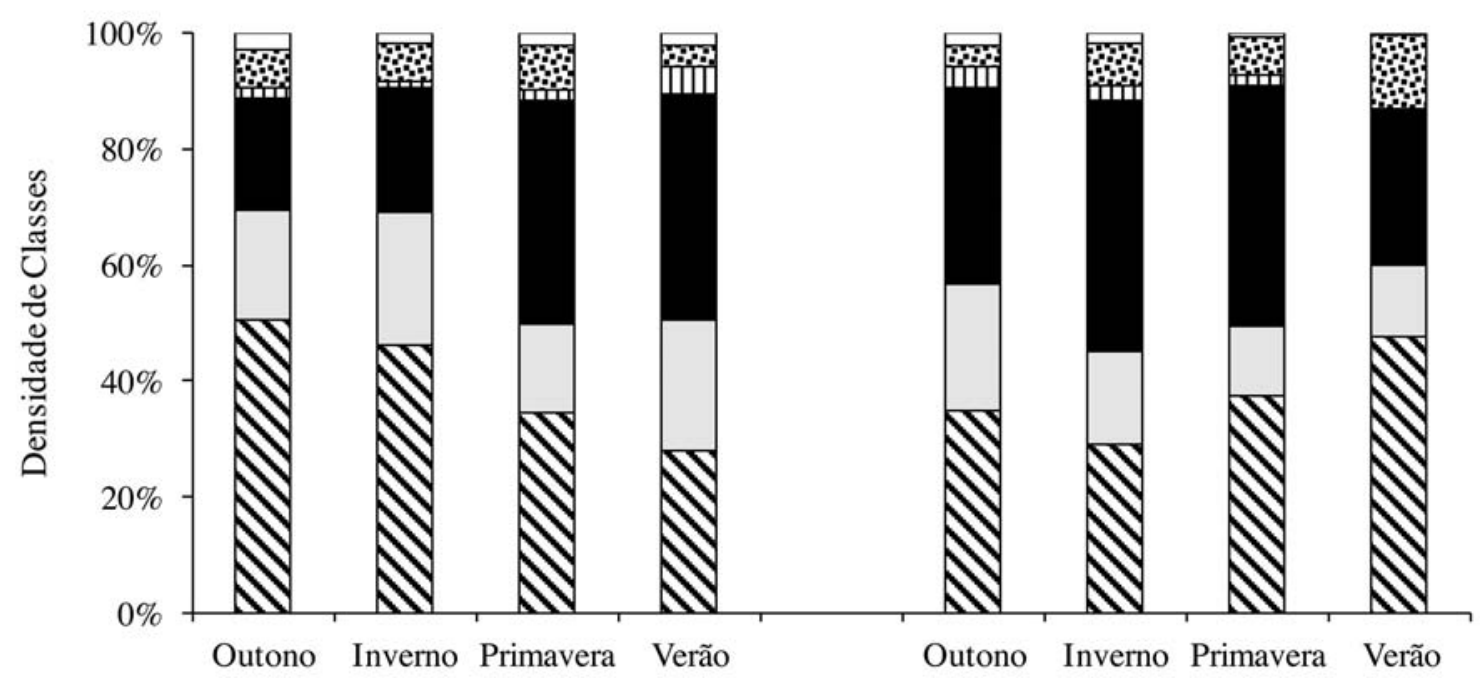

\begin{tabular}{|c|c|c|}
\hline $\begin{array}{l}\mathbf{\Delta B a c i l l a r i o p h y c e a e} \\
\mathbb{} \mathbf{C} \text { yanophyceace }\end{array}$ & $\begin{array}{l}\square \text { Chlorophyceae } \\
\text { Zygnemaphyceae }\end{array}$ & - Chrysophyceae \\
\hline
\end{tabular}

Figura 3 - Densidade relativa das classes algais no perifíton em Panicum repens nos pontos com presença de 3 e 4 espécies de macrófitas aquáticas nas estações do ano (3M: $\mathrm{n}=3 ; 4 \mathrm{M}: \mathrm{n}=2$, respectivamente).

Figure 3 - Relative density of periphytic algal classes on Panicum repens at sites with 3 and 4 macrophytes species in seasons (3M: $n$ $=3 ; 4 \mathrm{M}: \mathrm{n}=2$, respectively).

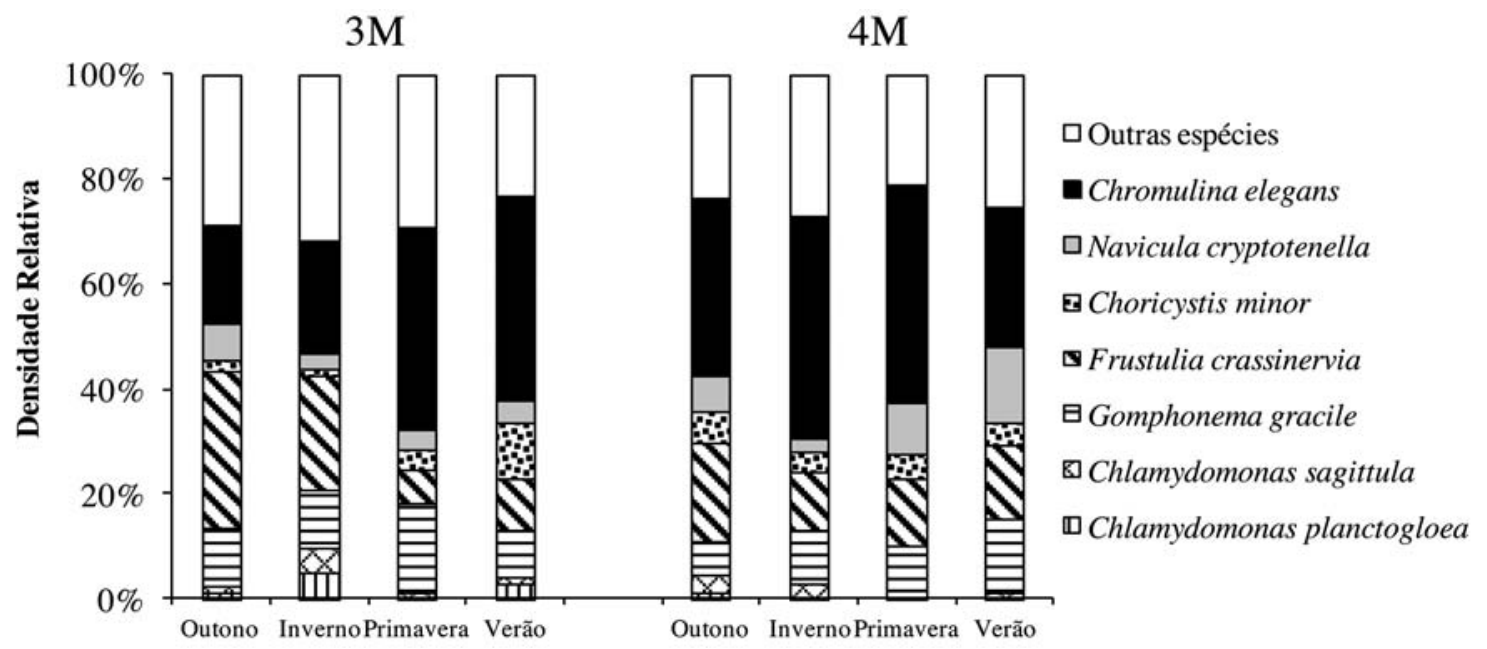

Figura 4 - Densidade relativa das espécies descritoras ( $>1 \%$ da densidade total) da comunidade de algas perifíticas em Panicum repens nos pontos com presença de 3 e 4 espécies de macrófitas nas estações do ano (3M: n = 3; 4M: $\mathrm{n}=2$, respectivamente).

Figure 4 - Relative density of the descriptors species ( $>1 \%$ of total density) of the periphyton community on Panicum repens at sites with 3 and 4 macrophytes species in seasons (3M: $n=3 ; 4 M: n=2$, respectively). 
A análise de correspondência canônica (CCA) realizada a partir de 33 espécies de algas perifíticas e cinco variáveis ambientais resumiu $20 \%$ da variabilidade total dos dados nos dois primeiros eixos (Fig. 5, Tab. 2). A correlação espécie-ambiente de Pearson para o eixo 1 ( $r=$ $0,889)$ e $2(r=0,790)$ indicou forte relação entre a distribuição das espécies e as variáveis ambientais. O teste Monte Carlo de randomização mostrou que os eixos 1 e 2 foram passíveis de interpretação ( $p$ $=0,05$ ).

O eixo 1 da CCA representou a variação sazonal das condições ambientais, ordenando pontos da primavera e do verão no lado positivo e do outono e inverno no lado negativo. As unidades amostrais da primavera e do verão foram mais correlacionadas com os altos valores de PDT e radiação subaquática $(r>0,6)$, enquanto as unidades do outono e do inverno foram mais correlacionadas com altos valores de NID e NT ( $r>-0,9)$. Com base na propriedade centroide, a ordenação indicou a maior associação de Ankistrodesmus fusiformis (Chlorophyceae) com os pontos de amostragem da primavera e de Encyonema mesianum (Bacillariophyceae) com os do verão, tendo ambas alta correlação com o eixo $1(r>-0,6)$. No lado negativo do eixo 1, Frustulia crassinervia (Bacillariophyceae), Cosmarium margaritatum e Cosmarium bioculatum (Zygnemaphyceae) apresentaram a maior correlação com o eixo e, ainda, observou-se a maior associação destas espécies com alguns pontos de amostragem do inverno. Os resultados da CCA indicaram que a sazonalidade apresentou forte influência sobre a composição das espécies descritoras da comunidade de algas perifíticas.

\section{Discussão}

Os resultados do presente estudo mostraram que a estrutura da comunidade de algas perifíticas em $P$. repens em pontos com presença de 3 e 4 espécies de macrófitas variou entre as estações do ano. Com base na densidade, biomassa algal e IA, evidenciou-se o maior desenvolvimento do perifíton no verão. Apesar da elevada cobertura de macrófitas nesta estação do ano, os pontos de amostragem caracterizaram-se pela maior transparência da água, radiação subaquática e concentração de fósforo dissolvido (PDT, $\mathrm{P}-\mathrm{PO}_{4}$ ), bem como pelo melhor balanço estequiométrico entre $\mathrm{N}$ e $\mathrm{P}$ (em média, $\mathrm{N}: \mathrm{P}=107$ ). Situação oposta foi observada no outono e no inverno, quando se detectou a maior participação dos componentes heterótrofos no perifíton. Estudos relataram que o aumento de transparência da água foi um importante fator ambiental para o desenvolvimento do perifíton em partes submersas de gramíneas e Panicum rivulare (Cetto et al. 2004; Martins \& Fernandes 2007). A maior disponibilidade de P na água foi, certamente, outro importante fator ambiental para o incremento de biomassa algal no Lago das Ninféias. Estudos observacionais e experimentais anteriores no reservatório evidenciaram o fósforo como fator limitante primário do perifíton e fitoplâncton (Fonseca \& Bicudo 2011; Fermino et al. 2011; Pellegrini \& Ferragut 2012). Apesar da alta variação sazonal na disponibilidade $\mathrm{N}$ na água, a concentração de nitrogênio inorgânico dissolvido foi sempre elevada (103-1602 $\mu \mathrm{g} \mathrm{L}^{-1}$, em média $730 \mu \mathrm{g} \mathrm{L}^{-1}$ ) e nenhum indício de limitação por $\mathrm{N}$ foi observado na água durante o período de estudo (razão molar N:P >65). Em relação ao número de espécies de macrófitas, estudos evidenciaram que a riqueza pode influenciar grandemente o acúmulo de biomassa e o estoque de fósforo no perifíton, afetando o funcionamento das áreas alagáveis (Engelhardt \& Ritchie 2001; McCormick et al. 2001). A influência da riqueza e da cobertura de macrófitas sobre a estrutura do perifíton foi relatada em outros estudos (Pellegrini 2012; Camargo \& Ferragut 2013). Contudo, os atributos estruturais da comunidade em $P$. repens não apresentaram clara relação com a riqueza e a cobertura total de macrófitas. A morfologia e a forma emergente de crescimento da macrófita podem contribuir para acúmulo do perifíton, independente da riqueza de macrófitas no ponto de amostragem.

Estudos relataram a formação de densas massas de algas em P. repens, sendo o crescimento algal de difícil controle nas áreas alagáveis da Flórida (Hanlon \& Langeland 2000; Hanlon \& Brady 2005). No reservatório do presente estudo, o pico de biomassa algal em $P$. repens foi cerca de 6 a 14 vezes maior do que o registrado em Nymphaea sp., Utricularia foliosa e Eleocharis acutangula (Pellegrini \& Ferragut 2012; Camargo \& Ferragut 2013; Santos et al. 2013). Recentemente, Rodusky et al. (2013) relataram, com base em 2 anos de estudo, que Panicum, considerada exótica no Lago Okeechobee (Flórida), acumulava muito mais biomassa perifítica do que a nativa Eleocharis celulosa. Assim ao que tudo indica, $P$. repens tem características morfológicas e/ou fisiológicas que parecem favorecer o incremento da biomassa perifítica. 


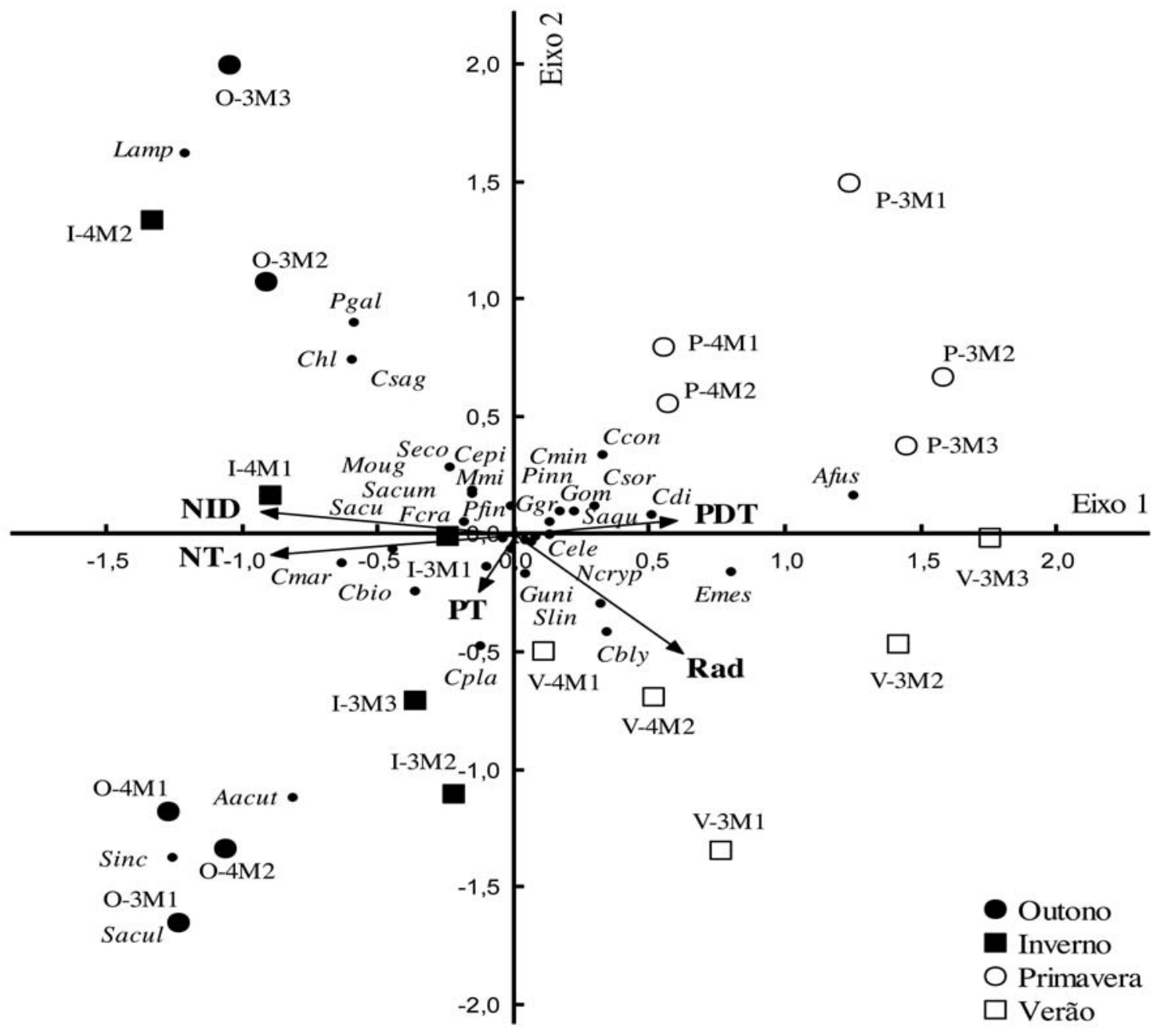

Figura 5 - Odernação pela CCA da densidade das espécies descritoras da comunidade de algas perifíticas em Panicum repens nos pontos com 3 e 4 espécies de macrófitas (3M e 4M, respectivamente) nas estações do ano. Abreviações das unidades amostrais: primeiro caractere refere-se à estação do ano (O-outono, I-inverno, P-primavera, V-verão) e os demais caracteres aos pontos de amostragem (3M e 4M). Abreviações dos vetores: NID nitrogênio inorgânico dissolvido, NT nitrogênio total, Rad radiação subaquática, PT fósforo total, PDT fósforo total dissolvido.

Figure 5 - CCA ordination of the descriptors species density of periphytic algal community on Panicum repens at sites with 3 and 4 macrophytes species (3M and 4M, respectively) in seasons. Abbreviations of sampling units: first character refers to the season (O-Autumn, I-Winter, P- Spring, V-Summer) and the others characters to the sampling sites (3M and 4M). Abbreviations vectors: NID dissolved inorganic nitrogen, total nitrogen NT, Rad subaquatic radiation, PT total phosphorus, total phosphorus dissolved PDT.

Apesar da variação da densidade de classes algais no perifíton ao longo do período de estudo, Bacillariophyceae e Chrysophyceae mantiveram a alta participação na estrutura da comunidade. Outros estudos também relataram elevada participação das diatomáceas no perifíton de partes submersas de gramíneas em reservatório eutrófico (Cetto et al. 2004) e de Panicum rivulare em lagoa impactada (Martins \& Fernandes 2007). Dentre as diatomáceas identificadas, Frustulia crassinervia, Gomphonema gracile e Navicula cryptotenella foram consideradas importantes descritoras da comunidade durante o período de estudo. Da mesma forma que em estudos anteriores no Lago das Ninféias (Pellegrini \& Ferragut 2012), F. crassinervia foi mais associada às condições ambientais do período seco. A diatomácea pedunculada G. gracile apresentou também elevada densidade relativa em todas as estações do ano. Esta espécie foi reportada 
Tabela 2 - Correlação de Pearson (r) entre a densidade das espécies descritoras da comunidade de algas perifíticas e os eixos 1 e 2 da CCA.

Table 2 - Pearson correlation (r) between species descriptors density of periphytic algae community and CCA axes 1 and 2.

\begin{tabular}{|c|c|c|c|}
\hline Táxons & Código & Eixo 1 & Eixo 2 \\
\hline Ankistrodesmus fusiformis Corda sensu Koršikov & Afus & 0,563 & 0,093 \\
\hline Chlamydomonas epibiotica H.Ettl & Cepi & $-0,313$ & 0,284 \\
\hline Chlamydomonas planctogloea Skuja & Cpla & $-0,129$ & $-0,305$ \\
\hline Chlamydomonas sagittula Skuja & Csag & $-0,435$ & $-0,295$ \\
\hline Chlamydomonas sordida Ettl & Csor & 0,154 & 0,098 \\
\hline Chlorococcales & Chl & $-0,366$ & 0,432 \\
\hline Choricystis minor (Skuja) Fott & Cmin & 0,338 & 0,278 \\
\hline Chromulina elegans Doflein & Cele & $-0,068$ & $-0,028$ \\
\hline Closterium dinae Ehr. ex Ralfs & Cdi & 0,340 & 0,084 \\
\hline Cosmarium bioculatum Brébisson ex Ralfs & Cbio & $-0,445$ & $-0,221$ \\
\hline Cosmarium blyttii Wille & Cbly & 0,318 & $-0,447$ \\
\hline Cosmarium contractum Kirchner var. minutum (Delponte) W.West \& G.S.West & Ccon & 0,220 & 0,318 \\
\hline Cosmarium margaritatum (Lundell) Roy \& Bisset & Cmar & $-0,593$ & $-0,083$ \\
\hline Gomphonema gracile Ehrenberg & Ggr & $-0,185$ & 0,017 \\
\hline Encyonema mesianum (Cholnoky) D.G.Mann & Emes & 0,570 & $-0,106$ \\
\hline Frustulia crassinervia (Brébisson) Lange-Bertalot \& Krammer & Fcra & $-0,442$ & $-0,175$ \\
\hline Geitlerinema unigranulatum (R.N.Singh) J.Komárek \& M.T.P.Azevedo & Guni & $-0,027$ & $-0,133$ \\
\hline Gomphonema sp. & Gom & 0,082 & 0,102 \\
\hline Lagynion ampullaceum (Stokes) Pascher & Lamp & $-0,406$ & 0,531 \\
\hline Monoraphidium minutum (Nägeli) Komárková-Legnerová & Mmi & $-0,047$ & 0,093 \\
\hline Mougeotia sp. & Moug & $-0,279$ & 0,276 \\
\hline Navicula cryptotenella Lange-Bertalot & Ncryp & 0,026 & 0,038 \\
\hline Pinnularia sp. & Pinn & 0,110 & 0,141 \\
\hline Pseudanabaena galeata Böcher & Pgal & $-0,312$ & 0,445 \\
\hline Pseudodidymocystis fina (Komárek) E.Hegewald \& Deason & Pfin & $-0,358$ & 0,028 \\
\hline Scenedesmus aculeolatus Reinsch & Sacul & $-0,288$ & $-0,363$ \\
\hline Scenedesmus acuminatus (Lagerheim) Chodat & Sacum & $-0,359$ & 0,109 \\
\hline Scenedesmus acunae Comas & Sacu & $-0,281$ & $-0,018$ \\
\hline Scenedesmus acutus Meyen var. acutus f. acutus & Aacut & $-0,270$ & $-0,337$ \\
\hline Scenedesmus ecornis (Ehrenberg) Chodat & Seco & $-0,312$ & 0,321 \\
\hline Scenedesmus incrassulatus Bohlin & Sinc & $-0,418$ & $-0,43$ \\
\hline Scenedesmus linearis Komárek & Slin & 0,094 & $-0,101$ \\
\hline Synechocystis aquatilis Sauvageau & Saqu & 0,157 & 0,072 \\
\hline
\end{tabular}


como descritora do perifíton em reservatório hipereutrófico (Borduqui \& Ferragut 2012) e mesotrófico (Pellegrini \& Ferragut 2012). Conforme a literatura, uma vantagem competitiva do Gomphonema é a aderência ao substrato por meio de pedúnculos, que permite a obtenção de P da água circundante (Burkholder \& Wetzel 1990; Passy 2007). No presente estudo, destacase, também, a elevada participação de Navicula cryptotenella no perifíton, principalmente nos pontos 4M da primavera e do verão. Esta espécie é considerada cosmopolita e de grande amplitude ambiental, sendo encontrada de ambientes oligotróficos a eutróficos (Salomoni et al. 2006; Schneck et al. 2008). N. cryptotenella foi descritora do perifíton em Nymphaea spp., sendo associada ao período de maior disponibilidade de nutrientes no reservatório de estudo (Pellegrini \& Ferragut 2012). Para Lane \& Brown (2007) as diatomáceas perifíticas em Panicum spp. constituem-se em boas indicadoras de impactos antrópicos nas áreas alagáveis.

Chrysophyceae foi, em média, a segunda classe de maior representatividade no perifiton em $P$. repens. Chromulina cf. elegans foi a espécie de maior densidade e frequência de ocorrência. Esta espécie é comumente citada como descritora no perifíton nos reservatórios rasos da área de estudo (Vercellino \& Bicudo 2006; Santos et al. 2013), sendo associada a alta disponibilidade de $\mathrm{P}$ na primavera (Fermino et al. 2011). De modo geral, as crisofíceas são excelentes competidoras, pois são oportunistas e capazes de alternar processos de autotrofia, heterotrofia e fagotrofia em respostas à condições ambientais (Sandgreen 1988).

De acordo com a análise de correspondência canônica (CCA), a sazonalidade foi grande fonte de variabilidade da densidade de espécies descritoras da comunidade de algas perifíticas. A concentração de nutrientes dissolvidos (NID e PDT) e de luz (radiação subaquática) foram as variáveis ambientais mais importantes na ordenação das unidades amostrais e espécies. Algumas espécies apresentaram forte associação com as estações do ano, como por exemplo, a Ankistrodesmus fusiformis na primavera, Encyonema mesianum no verão e algumas espécies de Scenedesmus no outono e inverno. Contudo, pouca ou nenhuma associação foi encontrada entre as espécies e os pontos com 3 ou 4 espécies de macrófitas. Este resultado mostrou que a presença de diferentes espécies não apresentou forte influência sobre a composição de espécies de algas perifíticas em $P$. repens.

As mudanças na estrutura do perifíton em $P$. repens foi determinada primariamente pela sazonalidade, tendo o número de espécies de macrófitas menor influência sobre a estrutura da comunidade no reservatório. Constatou-se que as condições ambientais do verão foram mais favoraveis ao desenvolvimento do perifíton em $P$. repens. Concluiu-se que a abundância, biomassa e composição de algas perifíticas mudaram fortemente entre as estações do ano e que, a disponibilidade de luz e de nutrientes, particularmente o fósforo, foram as variáveis ambientais mais associadas às mudanças estruturais da comunidade durante o período de estudo.Em decorrência do potencial invasor $P$. repens nos ecossistemas aquáticos tropicais (Pott \& Pott 2003; Agostinho et al. 2005), mais estudos serão necessários para o melhor o entendimento do complexo perifíton-Panicum repens.-

\section{Agradecimentos}

À FAPESP (Fundação de Amparo à Pesquisa do Estado de São Paulo), a concessão da bolsa de Iniciação Científica de MC (Processo 2010/074897) e o suporte financeiro para o desenvolvimento do projeto coordenado por CF (Processo 2009/522534). Ao MSc. Rodrigo Rodrigues Sampaio do Núcleo de Pesquisas Curadoria do Herbário do Instituto de Botânica de São Paulo - SMA, a identificação da macrófita Panicum repens. Os autores agradecem também à todos os estudantes e técnicos envolvidos no trabalho laboratorial e de campo.

\section{Referências}

Algarte, V.M.; Moresco, C. \& Rodrigues, L. 2006. Algas do perifíton de distintos ambientes na planície de inundação do alto rio Paraná. Acta Scientiarum. Biological Sciences 28: 243-251.

Agostinho, A.A.; Thomaz, S.M. \& Gomes, L.C. 2005. Conservação da biodiversidade em águas continentais do Brasil. Megadiversidade 1: 70-78.

APHA. 2005. Standard Methods for the Examination of Water and Wastewater. $21^{\text {st }}$ ed. American Public Health Association, Washington, DC. 1200p.Araújo, A. \& Bicudo, C.E.M. 2006. Criptógamos do Parque Estadual das Fontes do Ipiranga, São Paulo, SP. Algas, 22: Zygnemaphyceae (gêneros Actinotaenium, Cosmarium e Heimansia). Hoehnea 33: 219-237.

Battarbee, R.W. 1986. Diatoms analysis. In: Berglund, B.E. (ed.). Handbook of Holocene Paleohydrology. Wiley, New York. Pp 527-570. 
Bicudo, C.E.M.; Carmo, C.F.; Bicudo, D.C., Henry, R., Pião, A.C.S., Santos, C.M. \& Lopes, M.R.M. 2002. Morfologia e morfometria de três reservatórios do PEFI. In: Bicudo, D.C., Forti, M.C. \& Bicudo, C.E.M. (orgs.). Parque Estadual das Fontes do Ipiranga: unidade de conservação ameaçada pela urbanização de São Paulo. Ed. Secretaria do Meio Ambiente do Estado de São Paulo, São Paulo. Pp. 141-158.

Bicudo, D.C.; Necchi Júnior, O. \& Chamixaes, C.B.1995. Periphyton studies in Brazil: present status and perspectives. In: Tundisi, J.G.; Bicudo, C.E.M. \& Matsumura-Tundisi, T. (eds.). Limnology in Brazil. Academia Brasileira de Ciências e Sociedade Brasileira de Limnologia, Rio de Janeiro. Pp. 37-58.

Bicudo, C.E.M. 2004. Criptógamos do Parque Estadual das Fontes do Ipiranga, São Paulo, SP. Algas, 18: Chlorophyceae (Volvocales). Revista Brasileira de Botânica 27: 85-102.

Bicudo, C.E.M., Bicudo, D.C., Ferragut, C., Lopes, M.R.M. \& Pires, P.R. 2003. Criptógamos do Parque estadual das Fontes do Ipiranga, São Paulo, SP. Algas, 17: Chrysophyceae. Hoehnea 30: 127-153.

Borduqui, M. \& Ferragut, C. 2012. Factors determining periphytic algae succession in a tropical hypereutrophic reservoir. Hydrobiologia 683: 109-122.

Burkholder, J.M. \& Wetzel, R.G. 1990. Epiphytic alkaline phosphatase on natural and artificial plants in an oligotrophic lake: re-evaluation of the role of macrophytes as a phosphorus source for epiphytes. Limnology and Oceanography 35: 736-747.

Burkholder, J.M. 1996. Interaction of benthic algae with their substrates. In: Stevenson, R.J.; Bothwell M.L. \& Lowe, R.L. (eds.). Algal Ecology: freshwater benthic ecosystems. Academic Press, San Diego. Pp. 253-298.

Camargo, V.M. \& Ferragut, C. 2014. Estrutura da comunidade de algas perifíticas em Eleocharis acutangula (Roxb.) Schult em reservatório tropical raso. Hoehnea 41: 31-40.

Cetto, J.M.; Leandrini, J.A.; Felisberto, A.S. \& Rodrigues, L. 2004. Comunidade de algas perifíticas no reservatório de Irai, Estado do Paraná, Brasil. Acta Scientiarum Biological Science 26: 1-7.

Engelhardt, K.A.M. \& Ritchie, M.E., 2001. Effects of macrophyte species richness on wetland ecosystem functioning and services. Nature 411: 687-689.

Erhard, D. \& Gross, E.M. 2006. Allelopathic activity of Elodea canadensis and Elodea nuttallii against epiphytes and phytoplankton. Aquatic Botany 85: 203-211.

European Committee for Standardization (ECS). 2003. Water quality: guidance standard for the routine sampling and pretreatment of benthic diatoms from rivers. BS EN 13946.
Fermino, F.S.; Bicudo, C.E.M \& Bicudo, D.C. 2011. Seasonal influence of nitrogen and phosphorus enrichment on the floristic composition of the algal periphytic community in a shallow tropical, mesotrophic reservoir (São Paulo, Brazil). Oecologia Australis 15: 476-493.

Fonseca, B.M. \& Bicudo, C.E.M. 2011. Phytoplankton seasonal and vertical variations in a tropical shallow reservoir with abundant macrophytes (Ninféias Pond, Brazil). Hydrobiologia 665: 229-245.

Golterman, H.L. \& Clymo, R.S. 1971. Methods for chemical analysis of freshwaters. Ed. Blackwell Scientific Publications, International Biological Programmer, Oxford. 166p.

Golterman, H.L.; Clymo, R.S. \& Ohmstad, M.A.M. 1978. Methods for physical and chemical analysis of freshwaters. Blackwell Scientific Publications, International Biological Program, Oxford. 213p.

Grimshaw, H.J.; Wetzel, R.G.; Brandenburn, M.; Segerblom, K.; Wenkert, G.A.; Marsh, L.J.; Charnetzky, W.; Haky, J.E. \& Carraher, C. 1997. Shading of periphyton communities by wetland emergent macrophytes: decoupling of algal photosynthesis from microbial nutrient retention. Archiv für Hydrobiologie 139: 17-27.

Hanlon, C.G. \& Langeland, K. 2000. Comparison of experimental strategies to control torpedograss. Journal of Aquatic Plant Management 38: 40-47.

Hanlon, C.G. \& Brady, M. 2005. Mapping the distribution of torpedograss and evaluating the effectiveness of torpedograss management activities in Lake Okeechobee, Florida. Journal of Aquatic Plant Management 43: 24-29.

Kiss, M.K.; Lakatos, G.; Borics, G.; Gidó, Z. \& Deák, C. 2003. Littoral macrophyte-periphyton complexes in two Hungarian shallow waters. Hydrobiologia 506-509: 541-548.

Lalonde, S. \& Downing, J.A. 1991. Epiphyton biomass is related to lake trophic status, depth, and macrophyte architecture. Canadian Journal of Fisheries and Aquatic Sciences 48: 2285-2291.

Lane, C.R. \& Brown, M.T. 2007. Diatoms as indicators of isolated herbaceous wetland condition in Florida, USA. Ecological Indicators 7: 521-540.

Larned, S.T. 2010. A prospectus for periphyton: recent and future ecological research. Journal of the North American Benthological Society 29: 182-206.

Liboriussen, L. \& Jeppesen, E. 2009. Periphyton biomass, potential production and respiration in a shallow lake during winter and spring. Hydrobiologia 632: 201-210.

Legendre P. \& Legendre L. 2012. Numerical Ecology. Elsevier. 1006p.

Mackeret, F.J.H.; Heron, J. \& Talling, J.F. 1978. Water analysis: some revised methods for limnologists. Cumbria: Freshwater Biological (Association. Scientific Publication 39). Ed. Wilson, Son Ltda, Kendall. 117p. 
Marker, A.F.H.; Nusch, H.; Rai, H. \& Riemann, B. 1980. The measurement of photosynthetic pigments in freshwaters and standardization of methods: conclusion and recommendations. Archiv für Hydrobiologie 14: 91-106.

Martins, F.C.O. \& Fernandes, V.O. 2007. Estrutura da comunidade de algas perifíticas em substrato natural da lagoa da Universidade Federal do Espírito Santo, Brasil. Neotropical Biology and Conservation 2: 11-20.

McCormick, P.V.; O’Dell, M.B.; Shuford III, R.B.E. \& Backus, J.G. 2001. Periphyton responses to experimental phosphorus enrichment in a subtropical wetland. Aquatic Botany 71: 119-139.

McCune, B. \& Mefford, M.J. 2011. PC-ORD Version 6.0. Multivariate Analysis of Ecological Data. MjM Software Design, Oregon. 28p.

Passy S.I. 2007. Diatom ecological guilds display distinct and predictable behavior along nutrient and disturbance gradients in running waters. Aquatic Botany 86: 171-178.

Pellegrini, B.G. 2012. Influência da heterogeneidade espacial sobre a estrutura e estado nutricional (C, N, P) da comunidade perifítica em substrato natural (Nymphaea spp.). Dissertação de Mestrado. Instituto de Botânica, São Paulo. 95p.

Pellegrini, B.G. \& Ferragut, C. 2012. Variação sazonal e sucessional da comunidade de algas perifíticas em substrato natural em um reservatório mesotrófico tropical. Acta Botanica Brasilica 26: 807-818.

Pott, V.J. \& Pott, A. 2003. Dinâmica da vegetação aquática do Pantanal. In: Thomaz, S.M. \& Bini, L.M (eds.). Ecologia e Manejo de Macrófitas Aquáticas. EDUEM, Maringá. Pp.145-162.

Rodusky A.J., Sharfstein B., Hanlon C.G. \& Donnelly K.A. 2013. Ecological attributes of a native and exotic emergent subtropical marsh community in Lake Okeechobee, Florida (USA). Wetlands Ecology and Management 21: 87-105.

Salomoni, S.E.; Rocha, O.; Callegaro, V.L. \& Lobo, E.A. 2006. Epilithic diatoms as indicators of water quality in the Gravataí river, Rio Grande do Sul, Brazil. Hydrobiologia 559: 233-246.

Sandgreen, C.D. 1988. The ecology of Chrysophyte flagellates: their growth and perennation strategies as freshwater phytoplankton. In: Sandgreen, C.D. (ed.). Growth and reproductive strategies of freshwater phytoplankton. Cambridge University Press, Cambridge. Pp.9-104.

Santos, T.R., Ferragut, C. \& Bicudo, C.E.M. 2013. Does macrophyte architecture influence periphyton? Relationships among Utricularia foliosa, periphyton assemblage structure and its nutrient (C, N, P) status. Hydrobiologia 714: 71-83.

Sartory, D.P. \& Grobbelaar, J.U. 1984. Extraction of chlorophyll a from freshwater phytoplankton for spectrophotometric analysis. Hydrobiologia 114: 177-187.

Schneck, F.; Torgan, L.C. \& Schwarzbold, A. 2007. Epilithic diatom community in a high altitude stream impacted by fish farming in southern Brazil. Acta Limnologica Brasiliensia 19: 341-355.

Schneck, F.; Torgan, L.C. \& Schwarzbold, A. 2008. Diatomáceas epilíticas em riacho de altitude no sul do Brasil. Rodriguésia 59: 325-338.

Solorzano, L. 1969. Determination of ammonia in natural waters by the phenolhypochlorite method. Limnology \& Oceanography 14: 799-801.

Stevenson, R.J. 1997. Scale-dependent determinants and consequences of benthic algal heterogeneity. Journal of North American Benthological Society 16: 248-262.

Strickland, J.D.H. \& Parsons, T.R. 1960. A manual of seawater analysis. Bulletin Fisheries Research Board of Canada 125: 1-185.

Vadeboncoeur, Y. \& Steinman, A.D. 2002. Periphyton function in lake ecosystems. The Scientific World Journal 20: 1-20.

Valderrama, G.C. 1981. The simultaneous analysis of total nitrogen and total phosphorus in natural waters. Marine Chemistry 10: 109-112.

Vercellino, I.S. \& Bicudo, D.C. 2006. Sucessão da comunidade de algas perifíticas em reservatório oligotrófico tropical (São Paulo, Brasil): Comparação entre período seco e chuvoso. Revista Brasileira de Botânica 29: 363-377.

Wetzel, R.G. 1990. Land-water interfaces: metabolic and limnological regulators. Internationale Vereinigung für Theoretische und Angewandte Limnologie 24: 6-24. 
